\title{
Envelope Analysis on Vibration Signals for Stator Winding Fault Early Detection in 3-Phase Induction Motors
}

\author{
Chao Jin ${ }^{1}$, Agusmian P. Ompusunggu ${ }^{2}$, Zongchang Liu ${ }^{1}$, Hossein D. Ardakani ${ }^{1}$, Fredrik Petré ${ }^{2}$, and Jay Lee ${ }^{1}$ \\ ${ }^{1}$ NSF I/UCRC Center for Intelligent Maintenance Systems (IMS), University of Cincinnati, Cincinnati, OH, 45221, USA \\ jinco@mail.uc.edu \\ liuzc@mail.uc.edu \\ davarihn@mail.uc.edu \\ jay.lee@uc.edu
}

${ }^{2}$ Flanders' Mechatronics Technology Centre (FMTC), A Division of Flanders' Make vzw, Heverlee (Leuven), 3001, Belgium

agusmian.ompusunggu@fmtc.be

frederik.petre@fmtc.be

\begin{abstract}
This paper brings up a novel method for detecting induction motor stator winding faults at an early stage. The contribution of the work comes from the delicate handling of motor vibration by applying envelope analysis, which makes it possible to capture electrical short-circuit signature in mechanical signals, even if the magnitude of the fault is fairly incipient. Conventional induction motor condition-based maintenance methods usually involve current and voltage measurements, which could be expensive to collect, and vibration-based analysis is often only capable of detecting the fault when it is already quite significant. In contrast, the solution presented in this study provides a refreshing perspective by applying time-synchronous averaging to remove the discrete frequency component, and amplitude demodulation to further enhance the signal with the help of kurtogram. Experimental results on a three-phase induction motor show that the method is also able to distinguish different fault severity levels.
\end{abstract}

\section{INTRODUCTION}

In various industrial applications, such as high-speed trains, electric vehicles, industrial robots, and machine tools, threephase induction motors are always the driving force and one of the key machines of the whole system. Even with scheduled maintenance practices, unexpected failures of induction motors could still occur in these systems which would lead to excessive downtime and large losses in terms of maintenance cost and lost revenue. Condition-based

Chao Jin et al. This is an open-access article distributed under the terms of the Creative Commons Attribution 3.0 United States License, which permits unrestricted use, distribution, and reproduction in any medium, provided the original author and source are credited. maintenance $(\mathrm{CBM})$ and predictive maintenance $(\mathrm{PdM})$ have been proven to be maintenance strategies that are able to reduce unscheduled downtime and maintenance cost (Jardine, Lin and Banjevic, 2006). In CBM, maintenance activities are not scheduled for machines merely according to history of maintenance records or predefined maintenance rules on the basis of experience and/or expert knowledge, but also based on the present health status of the machines from sensory data, so that the waste owing to redundant maintenance and failures will be avoided. Such maintenance strategies require the integration of the following technologies: (a) on-line condition monitoring, (b) fault detection and diagnostics, and (c) prognostics.

Before designing any CBM or PdM strategies, common failure modes and effects analysis need to be conducted in order to identify the critical components and related issues. Figure 1 shows an industry survey result on the statistical distribution of failure modes typically occurring in squirrelcage induction motors (Bell, McWilliams, O'Donnell, Singh and Wells, 1985). It can be seen that rolling-element bearing

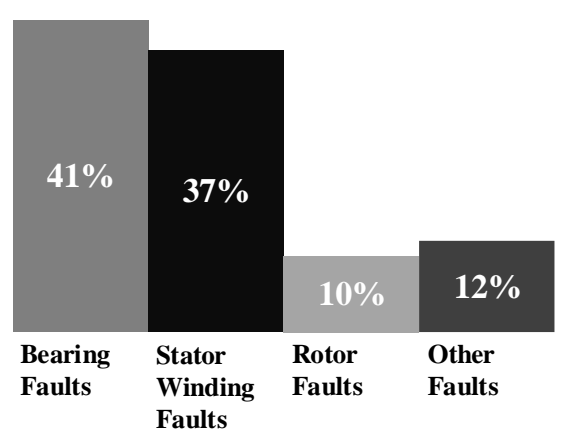

Figure 1. Statistics of failure modes in induction motors 
and stator winding failures due to insulation degradation contributes to almost $80 \%$ of the causes for unexpected breakdown in induction motors (Jover Rodríguez \& Arkkio, 2008). For rotary machinery in general, condition monitoring, diagnostics and prognostics for rolling-element bearings have been well studied during the past four decades due to its wide applications in almost all the rotary machinery (Lin \& Qu, 2000; Randall \& Antoni, 2011; Siegel, Al-Atat, Shauche, Liao, Snyder and Lee, 2012; Siegel, Ly and Lee, 2012).

For induction motor bearings in particular, vibration-based and motor current signature analysis based monitoring methods for rolling-element bearings in induction motors have been widely published in literature (Kliman \& Stein, 1992; Nandi, Toliyat and Li, 2005). However, the condition monitoring for winding insulation faults, especially vibration-based diagnostic and prognostic methods remain limited.

Winding faults due to stator coil degradation can be classified into four categories (Ukil, Chen and Andenna, 2011), namely (a) inter-turn short of the same phase, (b) short between coils of same phase, (c) short between two phases, and (d) short between phase to earth. Among them, inter-turn faults are considered to be the most challenging type of winding faults to be detected in induction motors. The online condition monitoring methods for motor winding fault detection are summarized in Figure 2. Most of the online monitoring methods are based on current and voltage signals, among which the symmetric component current balance monitoring (Furfari \& Brittain, 2002; Eftekhari, Moallem, Sadri and Hsieh, 2013; Ompusunggu, Liu, Ardakani, Jin, Petre and Lee, 2014), negative sequence impedance detector (Kliman, Premerlani, Koegl and Hoeweler, 1996; Kohler, Sottile and Trutt, 2002), voltage mismatch (Sottile, Trutt and Kohler, 2000; Trutt, Sottile and Kohler, 2002), and Parks vector (Cardoso, Cruz and Fonseca, 1999) are the most widely referred methods. Nevertheless, these methods require the measurements of 3-phase current or voltage signal from induction motors, which involves expensive sensors and data acquisition (DAQ) hardware. Furthermore, direct measurements of 3-phase voltages from motor windings are not feasible for online application, and the voltage measurements from the frequency-inverter drive are usually pulse-width modulation (PWM) signals that need additional signal processing efforts.

Compared with the current and voltage-based winding fault monitoring, vibration-based methods have the advantages of (a) requiring less expensive sensors, (b) requiring less channels for the DAQ system, and (c) monitoring mechanical failures at the same time.

As a result, vibration-based monitoring for induction motors has been gaining popularity. Similar to mechanical faults, induction motor stator winding faults also generate

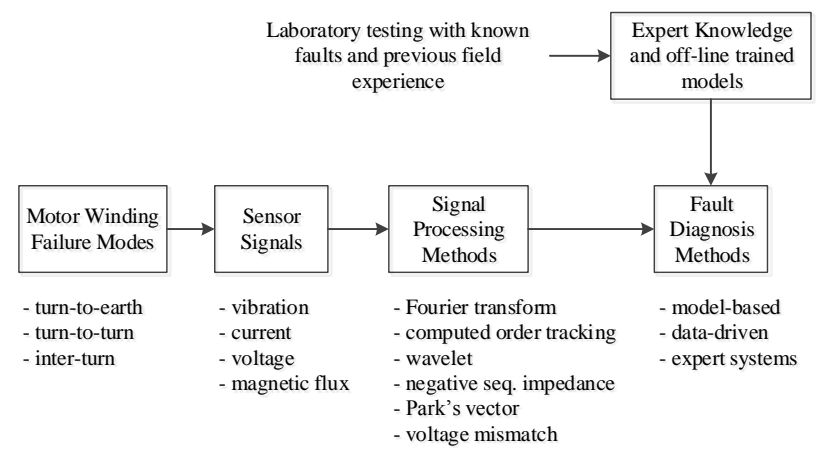

Figure 2. Online condition monitoring methods for motor winding fault (Sin, Soong and Ertugrul, 2003)

additional magnetomotive forces that change the pattern of motor vibration. Lamin et al (2014) found that these pattern changes are usually reflected in the frequency domain at harmonics of slot frequency and supply frequency. Nevertheless, these features are not significant enough to be detected unless the faulty turns are around $5 \%$ of the total windings (Lamim, Brito, Silva and Pederiva, 2013). Besides, it is required that not just vibration signals but also magnetic flux signals as an essential complement to determine the diagnostic frequencies.

Djurović et al (2014) proposed a method that utilizes a torque signal physical model to locate the interested frequencies and detect the faults at the same frequency ranges in the vibration data. The method was able to differentiate unbalanced supply from stator winding faults. Yet it still requires torque signals for the physical modeling process to determine the characteristic frequencies and detect the winding faults in the stator.

Seshadrinath et al (2014) used complex wavelets to extract vibration signatures that were able to distinguish inter-turn faults from mechanical faults such as bearing damage. The performance was proved to be better than the traditional discrete wavelet transform. However, wavelet transform is relatively computationally expensive, and the correct setting of the number of levels to decompose usually calls for tuning.

This paper proposes a combination of different signal processing techniques to mine and amplify the motor winding fault related features solely based on vibration signals and tachometer signals. The advantages of the proposed method include:

- The method does not require torque signal or magnetomotive flux signal to build a physical model to determine diagnostic frequency of the vibration signal. 
- The fault detection process can be much more automated and can be applied by users with minimum domain knowledge.

- As a data-driven method, there is also less parameters to tune and less computationally complex compared to other peer methods.

The focus of this research was neither to differentiate winding faults from mechanical faults, nor tell the difference between unbalanced stator winding from unbalanced supply, but to explore the possibility of detecting stator inter-turn faults at a very early stage, and try to find an automatic way to determine the frequency ranges that contain diagnostic information. Time synchronous averaging, spectral kurtosis filtering, and envelope analysis have been implemented in the signal processing process. As will be discussed in the results section, the first order of envelope spectrum shows monotonically increasing trend as the level of winding insulation degradation increases.

The remaining part of the paper is organized as follows: Section 2 discusses about the methodology development and theoretical background of the signal processing techniques applied to the motor vibration signals; Section 3 briefly introduces the experimental setup and the test procedure for data generation; Section 4 demonstrates the effectiveness of the proposed vibration signal processing methods and the selected features through the experimental data analysis; and Section 5 summarizes the important findings obtained in this study.

\section{METHODOLOGY DEVELOPMENT}

\subsection{Method Overview}

Vibration-based signal processing has long been favored for the diagnostics and prognostics of mechanical faults in rotary machinery, such as rolling-element bearings and gearboxes (Al-Atat, Siegel and Lee, 2011; Randall \& Antoni, 2011). Depending on the physics of faults, some mechanical faults such as unbalance and gear eccentricity manifest periodic vibration signature. Other type of faults assume that the concerned fault would lead to transients and impulses in vibration signals, which do not occur in the nominal state.

Inspired by the rolling-element bearing fault diagnosis (Randall \& Antoni, 2011), this paper addresses the issue of detecting induction motor stator inter-turn faults when they are still preliminary. Detection of the impulses hidden in the smearing and noise calls for appropriate signal processing techniques to diminish the effect of deterministic vibration patterns generated by mechanical structure, and emphasize the faulty characteristics buried in the remaining random part (Randall \& Antoni, 2011). It is assumed that stator winding fault will cause disturbance in the magnetic field which might be taken as random variation in regular frequency analyses of the raw signal. The induction motor vibration signal is then assumed to be cyclostationary and the winding fault diagnostic information can be detected using envelop analysis. As shown in Figure 3, the first step of signal processing is to check the quality of the vibration data (Jabłoński, Barszcz and Bielecka, 2011; Jablonski \& Barszcz, 2013) to guarantee data integrity. A list of statistics including mean and root-mean-square is calculated and compared with a set of predefined thresholds. Then, the vibration data that have passed the data quality check will go through a low-pass filter to exclude the high frequency noise (In this study, the cut-off frequency was set to be one half of Nyquist frequency, which is $12800 \mathrm{~Hz}$ for the vibration signal, and $10 \mathrm{~Hz}$ for the tachometer signal, since the ratio of tachometer is 1/4.) The cut-off frequency of the vibration signal is determined based on visual inspection of the vibration signal spectrum. Up to 0.8 of Nyquist frequency was checked, and neither characteristic peaks nor resonance peaks were observed at higher frequency range. The peaks at higher frequencies were not very consistent which made us believe that they are more like noise of DAQ and should be filtered. After the aforementioned pre-processing steps, time synchronous averaging is performed to calculate the discrete frequency component (Randall \& Antoni, 2011) caused mainly by motor pulley. This deterministic component in the signal is then subtracted from the raw signal, and the remaining residual signal will only contain the random part, which carry the features from electrical faults. Furthermore, the resonance frequency band that amplifies the fault will be located via spectral kurtosis analysis and kurtogram. The output center frequency and bandwidth will be referred to for the subsequent envelope analysis. Eventually, the first order component of the envelope spectrum is found to be an effective health index.

The following sub-sections will introduce the theory of the tools and techniques utilized in this work and explaining why they are effective in detecting stator inter-turn faults in induction motors. For each technique introduced, some of the results from the later experimental research will be presented accordingly for a better explanation and clarity.

\subsection{Theoretical Background}

Instead of going through the calculation of magnetic forces, the induction motor winding fault detection strategy is formulated from the perspective of vibration signal processing.

To state mathematically, the problem is to extract the stator inter-turn faulty signal $x(t)$ buried in the noise $\eta(t)$. And the actual raw signal $s(t)$ collected from the motor is the combination of the two, which is (Antoni \& Randall, 2006)

$$
s(t)=x(t)+\eta(t)
$$

Under this problem statement, the following assumptions for this research are proposed: 


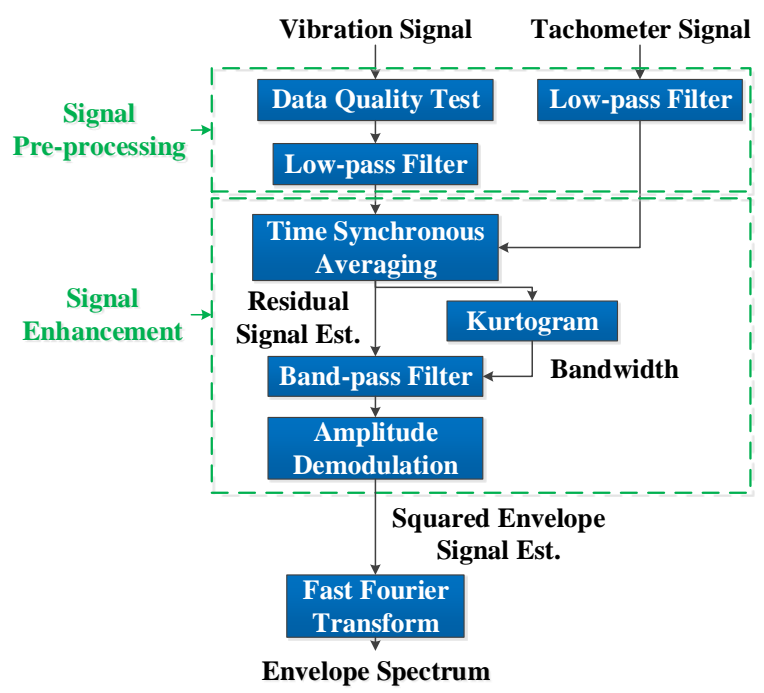

Figure 3. Flowchart of inter-turn fault detection for threephase induction motors using vibration signal.

1. The inter-turn faulty signal $x(t)$ has transients and contains impulses, which do not occur, or follow a different pattern in the healthy conditions;

2. The noise $\eta(t)$ refers to not only the stationary measurement noise, but also the discrete frequency component, namely the vibration influence from the mechanical elements such as shaft and geared pulley.

\subsubsection{Time synchronous averaging}

Time synchronous averaging (TSA) is an essential tool for rotating machines that extracts periodic waveforms from noisy data. TSA is performed with respect to a certain shaft according to the tachometer signal as angular position reference. Vibration signals that went through TSA process will have an integer number of orders of the fundamental harmonic (shaft frequency) retained, and other nonsynchronous vibration components weakened. If the synchronous-averaged signal is subtracted from the original signal, the residual signal with the harmonics of the shaft frequency removed will be obtained. Both the synchronousaveraged signal and residual signal contain diagnostic information of different failure mode (Al-Atat et al., 2011). While there are many different techniques for TSA, zero crossing-based technique is the most widely used method.

Zero crossing-based TSA resamples the vibration signal to angular domain where the samples recorded in one shaft rotation are interpolated into a fixed number of data points for each revolution. The number of points per revolution $N$ is derived from Eq. (2):

$$
N=2^{\text {ceiling }\left(\log _{2} \max (n)\right)}
$$

where $n$ is the number of points between two subsequent zero crossing indices of the tachometer signal (Bechhoefer \&
Kingsley, 2009), and "ceiling" represents the process of rounding toward positive infinity.

However, it should be noted that resampling from time domain to angular domain will cause problems for the following signal processing steps since the kernel functions of kurtogram, filtering, and envelope analysis have a constant frequency $(\Delta t)$ instead of constant angle $(\Delta \vartheta)$. Hence the synchronous-averaged signal should be interpolated back to its original time-based sampling mechanism before calculating the residual signal.

The procedure of obtaining the residual signal from TSA is summarized as follows:

(1) Find zero-crossing indices in the tachometer signal and calculate the zero crossing time (ZCT) with interpolation.

(2) For each ZCT, calculate the time between $\mathrm{ZTC}_{k}$ and $\mathrm{ZCT}_{\mathrm{k}+1}$, namely, $\mathrm{dZCT}_{\mathrm{k}}$, where $k$ is the crossing point index.

(3) Calculate the resampled time interval: $d Z C T / N$, where $N$ is given by Eq. (2). Interpolate the signal to the newly resampled time and accumulate the resampled data.

(4) Save the original time stamps for each revolution.

(5) Repeat step (2) through (4) for all the revolutions, and then divide the accumulated $N$ point vector by number of revolutions.

(6) Interpolate the $N$ point vector (TSA signal) back to the original time stamps for each revolution, and combine the interpolated TSA signal to get the same length of vector as the original data.

(a)

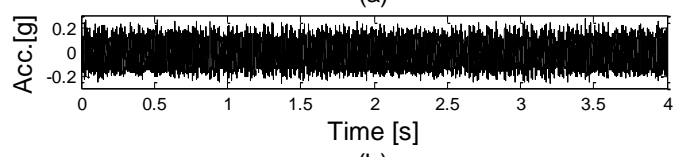

(b)

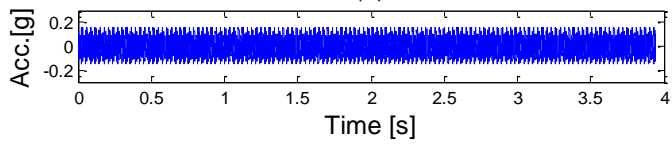

(c)

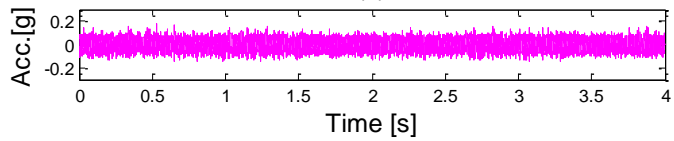

Figure 4. Time domain signal result for TSA analysis. (a) time domain raw signal with kurtosis of 2.3262, (b) time domain TSA signal, (c) time domain residual signal with kurtosis of 3.0590. The induction motor was running at approximately $2000 \mathrm{rpm}$ and an inter-turn fault was induced. 


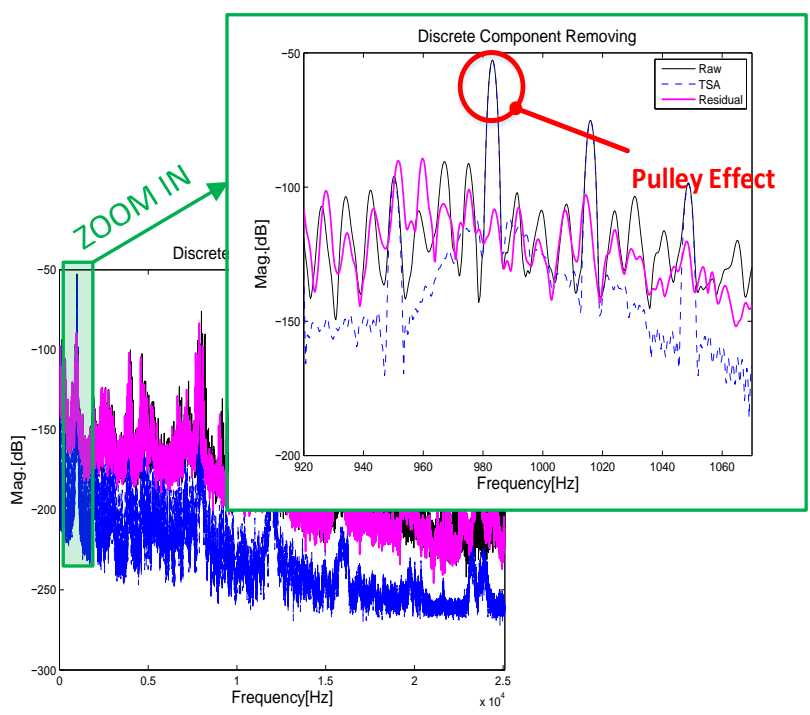

Figure 5. Power spectrum density comparison between raw signal, TSA signal, and residual signal of the TSA analysis. The induction motor was running at approximately $2000 \mathrm{rpm}$ $(33.3 \mathrm{~Hz})$ with inter-turn fault induced. The number of teeth on the pulley attached to the motor is 30 . The circled peak in PSD plot is located at around 983.2 Hz.

(7) Subtract the combined vector from the original data to get the residual signal.

An example of TSA analysis is shown in Figure 4 and Figure 5. In Figure 4, it could be hard to tell the difference between the residual signal and the original one, except that the amplitude level seem to be decreased. Nevertheless, if we compare the kurtosis of the original signal, which is 2.3262 , with the kurtosis of the residual signal, which is 3.0690 , it is evident that the transients in the signal has been amplified. To reason the mechanism of TSA analysis, power spectrum density (PSD) plot is provided in Figure 5. It can be observed that the main component (highest peak) of TSA signal PSD locates at around $983.2 \mathrm{~Hz}$, which is approximately 30 times of the revolutionary frequency of the induction motor rotating at $2000 \mathrm{rpm}$. This coincides with the fact that the motor has a 30-teech pulley as its component. This confirms the success of our intension to remove the contamination of deterministic component generated by mechanical components.

\subsubsection{Spectral kurtosis and kurtogram}

Kurtosis as a statistical feature is well-known as a global value to detect the peakiness in a signal. It is defined as

$$
k=\frac{E\left[(x(t)-E[x(t)])^{4}\right]}{E\left[(x(t)-E[x(t)])^{2}\right]^{2}}
$$

where $E[\bullet]$ indicates the statistical expectation. Spectral kurtosis is an extension of kurtosis to a function of frequency, and is known for identifying the impulsiveness in the frequency spectrum for rotary machinery fault diagnosis. It is calculated based on the short-time-Fourier-transform $X(t, f)$ of the original signal. As mentioned by Randall \& Antoni in (Randall \& Antoni, 2011), spectral kurtosis is defined as

$$
K(f)=\frac{E\left[(X(t, f)-E[X(t, f)])^{4}\right]}{E\left[(X(t, f)-E[X(t, f)])^{2}\right]^{2}}-2
$$

The benefit of spectral kurtosis analysis is that it is able to find a frequency band that contains fault characteristics without requiring a large number of history data. Yet it is then of vital importance that an appropriate window length be chosen for the short-time-Fourier-transform. In order to find the optimal window length, or equivalently bandwidth, more efficiently, fast kurtogram was adopted to plot spectral kurtosis against level and frequency. Fast kurtogram is a "suboptimal" version of kurtogram with poorer resolution but much less computational pressure. Similar to discrete wavelet packet transform, starting from the largest bandwidth (spectrum), it iteratively divides the spectrum into two equal parts and form a tree-structure at the end (Antoni, 2007). Another task for kurtogram is to find the center frequency with the highest spectral kurtosis value, which is related to the resonance frequency of the motor. The incipient vibration that was caused by stator winding fault will be amplified at this resonance frequency. It can be observed in Figure 6 that the color in the fast kurtogram indicates the value of kurtosis, and in this particular example the highest kurtosis exists at Level 5.5 with a center frequency of around $12000 \mathrm{~Hz}$. Even though the fast kurtogram computes automatically the center frequency and the bandwidth, the original power spectrum density still needs to be taken into consideration to finalize the spectrum section that needs to be demodulated later. This part will be shown with graphical explanation in the following sub-section.

\subsubsection{Envelope Analysis}

Often, the spectrum of raw vibration signal for rotary machinery gives little insight on faulty characteristics due to excessive environmental and structure noise. As mentioned in previous sections, winding faults at early stage would induce mechanical impacts that are amplified at the resonance frequency of the induction motor system. With kurtogram analysis, envelope analysis will further improve the signal to noise ratio and enhance the transients, so that the fault signature can become more pronounced.

The procedure for envelope analysis in this research is described in Figure 7, where the residual signal estimation 


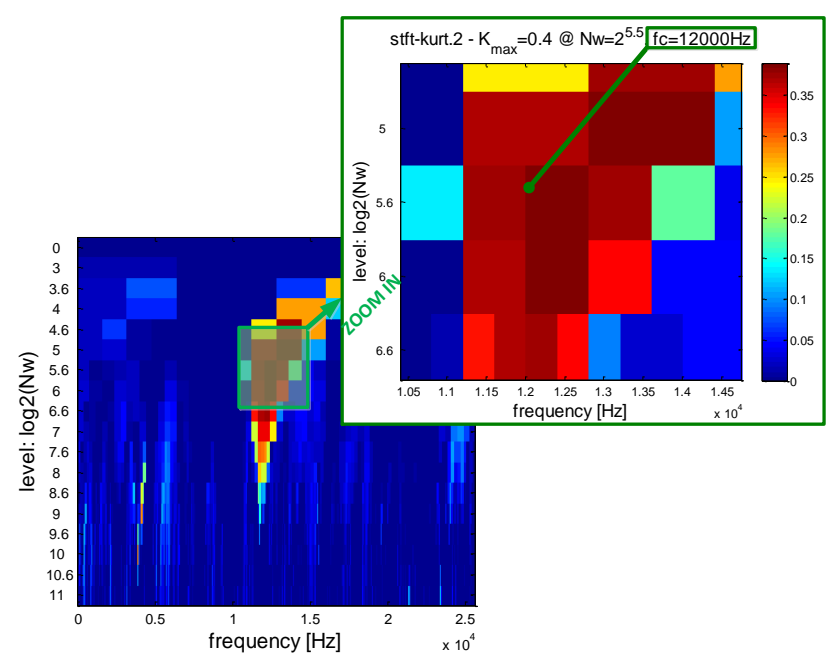

Figure 6. Kutogram of inter-turn fault residual signal at 2000 rpm. The highest kurtosis is 0.4 at Level 5.5 with a center frequency of $12000 \mathrm{~Hz}$.

from the TSA process is the input and the envelope spectrum is the output. First, a band-pass filter is designed based on the center frequency and bandwidth determined from fast kurtogram.

Then the resulting band-passed residual signal is modulated with a carrier frequency the same as the center frequency. Specifically, it is calculated by following Eq. (5).

$$
y(t)=r(t) \times \exp \left(-j 2 \pi f_{c} t\right)
$$

where $r(t)$ is the band-passed residual signal estimation calculated from the TSA, $j=\sqrt{-1}, f_{c}$ is the center frequency, and $y(t)$ is the modulated signal. The purpose of the first step is to shift the interested frequency band to the DC frequency, with $f_{c}$ overlapping with the DC component. Afterwards, the modulated signal is processed through a low-pass filter with half of the bandwidth as the cutoff frequency. The obtained narrow-band complex signal is represented by $\tilde{y}(t)$, whose amplitude is the estimated envelope. Eventually, $\tilde{y}(t)$ will be multiplied by its complex conjugate $\tilde{y}^{*}(t)$ to get the squared envelope, which is calculated by following Eq. (6):

$$
e(t)=\tilde{y}(t) \times \tilde{y}^{*}(t)
$$

where $e(t)$ represents the squared envelope signal.

The result of demodulation can be found in Figure 8. In the time domain, the emphasis of impulsiveness in the faulty signal can be recognized even by observation. Quantitatively, the kurtosis of the signal has increased from 3.0590 to 4.2691. In the frequency domain, one can clearly see in Figure 8 (b) that the peaky section centered at approx. $12000 \mathrm{~Hz}$ with a bandwidth of around $800 \mathrm{~Hz}$ is highlighted. This is where the high frequency band that contains the faulty information locates. It was picked up by

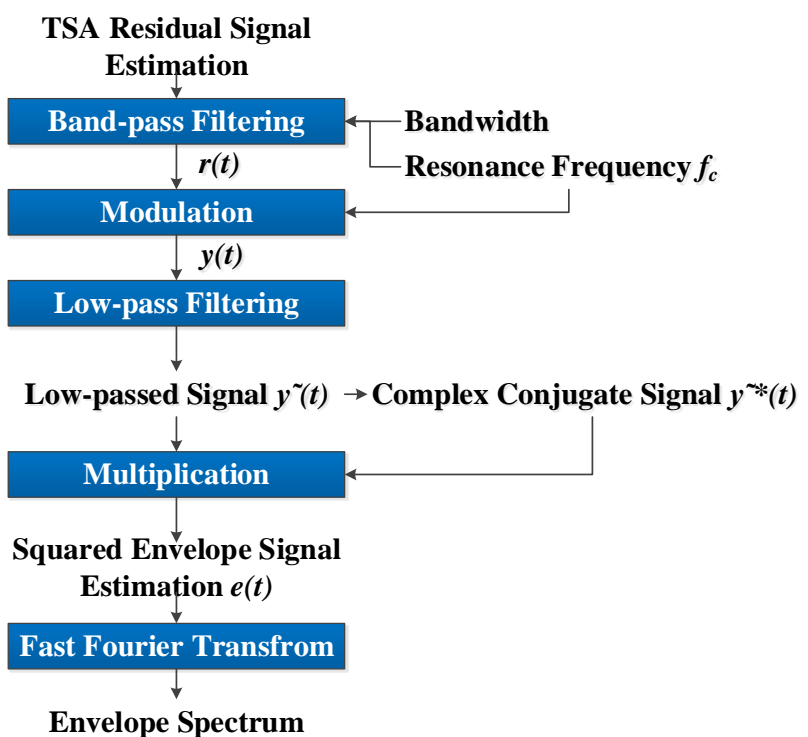

Figure 7. Flowchart of envelope analysis. The resonance frequency (center frequency) and bandwidth are determined with the help of kurtogram. Signals are represented by the same symbols used in the text description. Since the blue box represents a process and borderless text represent a signal, some symbols are indicated right beside the text, instead of beside the arrow.

kurtogram and moved to lower frequency band after demodulation. Discussion on the result of envelope signal and envelope spectrum will be formulated in Section 4.

\section{EXPERIMENTAL SETUP}

For conducting this research, a dedicated induction motor test-bed was designed and developed (Ompusunggu et al., 2014). The test-bed is designed such that one is able to simulate the winding faults with different levels of severity and collect vibration, current, voltage and torque signals from the motor. The winding faults that could be induced in the system include (i) inter-turn and (ii) turn-to-earth faults. The test-bed was also designed to run at different speed regimes and load conditions for multi-regime data collection and analysis. The following sections will briefly describe the testbed design, the procedure for inducing winding faults and the experiments with different fault conditions.

\subsection{Test Setup}

The test-bed consisted of an 11KW, 19.7A, 400V 3-phase induction motor driven by a variable frequency drive. The rotational speed of the motor could be varied from 0 to 3000 RPM with both stationary and transient modes available. A magnetic brake was connected to the output shaft of the motor through a timing-belt and pulley mechanism. The mechanism allowed the brake shaft to rotate at half of the speed of the motor shaft. By controlling the input current of the brake, an external load varying from 0 to $50 \mathrm{Nm}$ could be 
applied to the motor. A PC with LabVIEW programs was used to send the control signals to the variable frequency drive and magnetic brake controller. A variable resistor with the range of $0-580 \Omega$ was used to simulate different levels of severities in the shorted turns in inter-turn faults. A tri-axial accelerometer was mounted on the top of the housing of the motor to collect the vibration of the motor. A tachometer based on a proximity probe was used to measure the rotational speed of the motor. The head of the tachometer was put towards a 4-tooth flywheel connected to the motor shaft generating 4 pulses per revolution. The experimental setup and the schematic view of the test-bed are shown in Figure 9 and Figure 10.

\subsection{Fault Simulation}

The winding of the motor used in the test-bed is randomwound (Figure 11). The winding was modified by connecting three shielded wires to the coil of phase $\mathrm{w}$ at three locations and the other ends of the wires were brought outside as schematically shown in Figure 12. The inter-turn faults were simulated by connecting the other ends of the wires to a variable resistor.

For healthy state simulation, the ends of the three wires were left unconnected. The inter-turn faults were simulated under two different scenarios referred to as inter-turn I and II. In inter-turn I, wires 1 (in orange) and 2 (in green) were connected through a variable resistor. Similarly for inter-turn II, wire 1 was shorted to wire 3 (black) through a variable resistor. By adjusting the resistance to 580 and $300 \Omega$, two levels of severity for both inter-turn I and II were simulated, as summarized in Table 1. These values were set up based on our third-party motor manufacturer's experience of typical resistance of inter-turn faults for such a motor.

\begin{tabular}{ccc}
\hline State & Resistance $[\Omega]$ & Comment \\
\hline F1 & 580 & Lowest level \\
\hline F2 & 300 & Moderate level \\
\hline
\end{tabular}

Table 1. Different fault levels for induction motor

\subsection{Test Procedure}

The test was performed at the constant speed of 2000 RPM and constant brake torque of $12 \mathrm{Nm}$ for all the winding conditions. At each level of winding faults, the current $i_{l}$ flowing through the variable resistor was measured and the corresponding dissipated power $P_{d}$ was calculated as summarized in Table 2. The exact same test settings were repeated in order to check the consistency. (a)

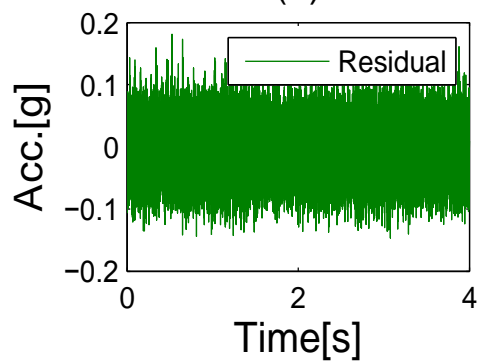

(d)

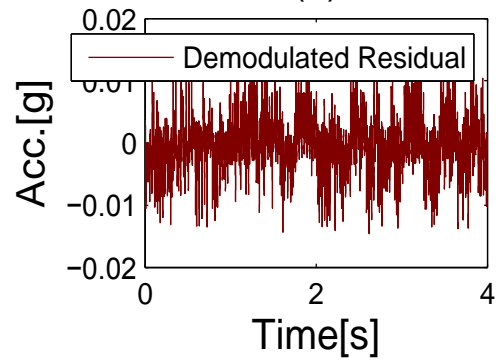

(b)

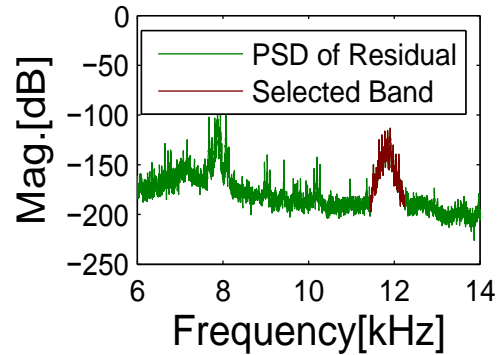

(e)

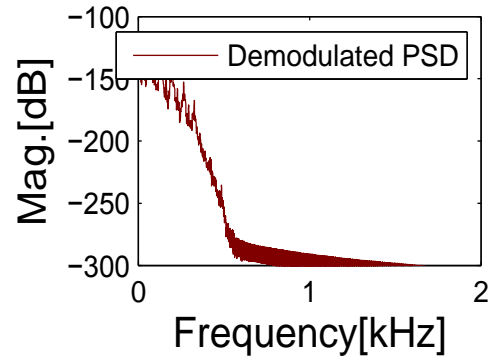

(c)

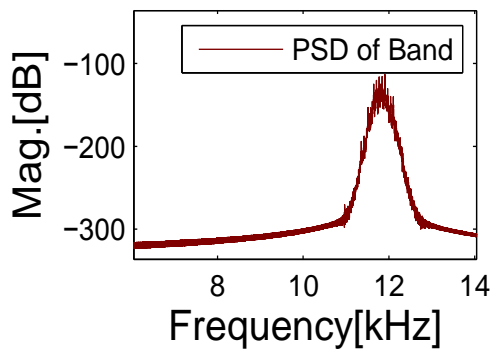

(f)

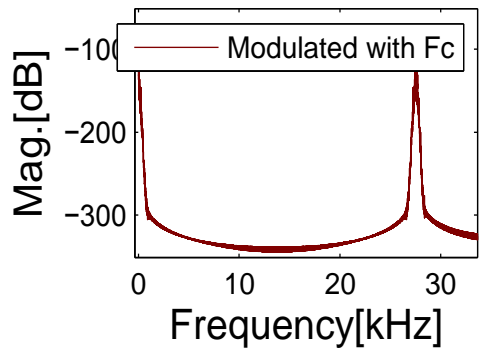

Figure 8. Comparison of time domain and frequency domain signal before and after demodulation: (a) time domain TSA residual signal estimate with kurtosis 3.0590, (b) Welch estimate power spectrum density of TSA residual with high-frequency band highlighted in dark red, (c) power spectrum density after band-pass filtering the selected resonance frequency band, (d) time domain envelope TSA residual signal with kurtosis 4.2691, (e) Welch estimate power spectrum density of the demodulated TSA residual, (f) power spectrum density after modulating the band-pass filtered signal with the center frequency $F_{c}$. The signal comes from the condition of inter-turn fault. Note that the scales of plots are different. 


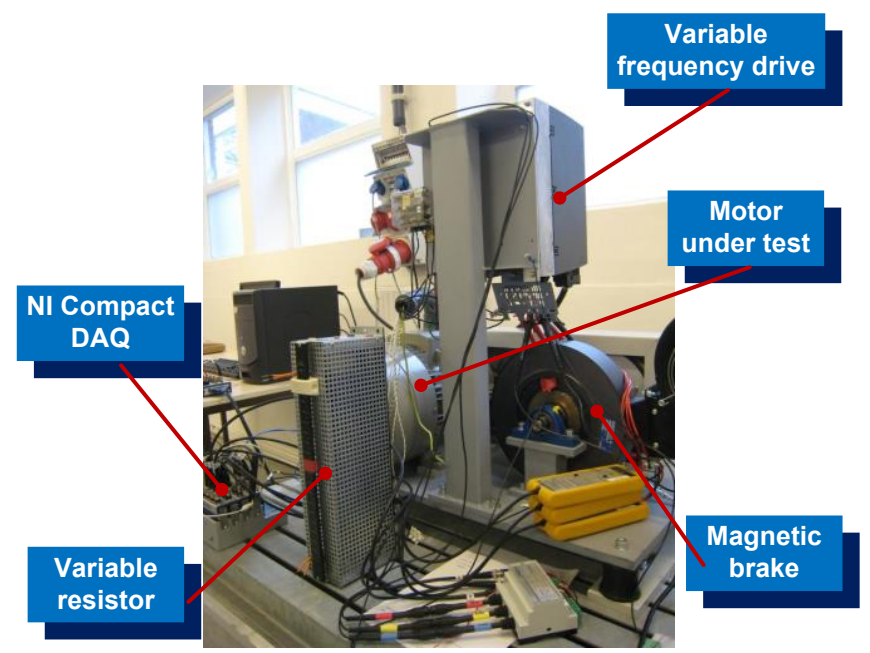

Figure 9. Photograph of the induction motor test bed.

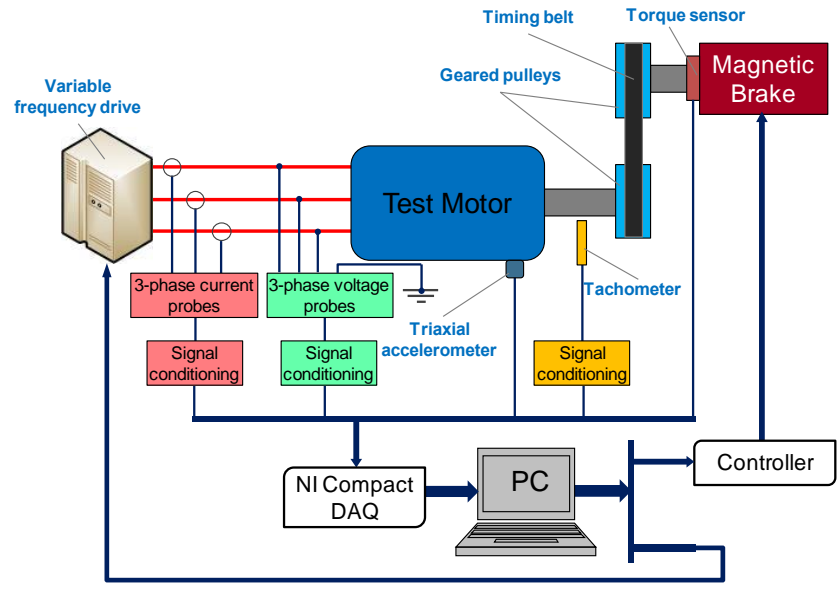

Figure 10. Schematic view of the motor test bed.

Prior to digitizing the signals, each measured signal was passed through a low-pass and an anti-aliasing filter embedded in each channel of the NI data acquisition system. Doing the tests in this way ensures that the potential aliasing problems caused by high frequency noise can be avoided. Depending on the sampling frequency, the cut-off frequency of the anti-aliasing filter was automatically adjusted. The vibration signals were sampled at the rate of $51.2 \mathrm{KHz}$ with the duration of four seconds. The duration of the signal mainly depends on the following two aspects: (a) the rotational speed of the motor (longer signal length at lower speeds); and (b) level of noise due to environmental, structural, and DAQ condition. Thus, there is no universal cutoff threshold for the duration, yet it is suggested that the data samples collected to be above $2^{11}$ to guarantee sufficient Fourier transform resolution. The digitized data was stored in the PC and analyzed off-line in MATLAB software.

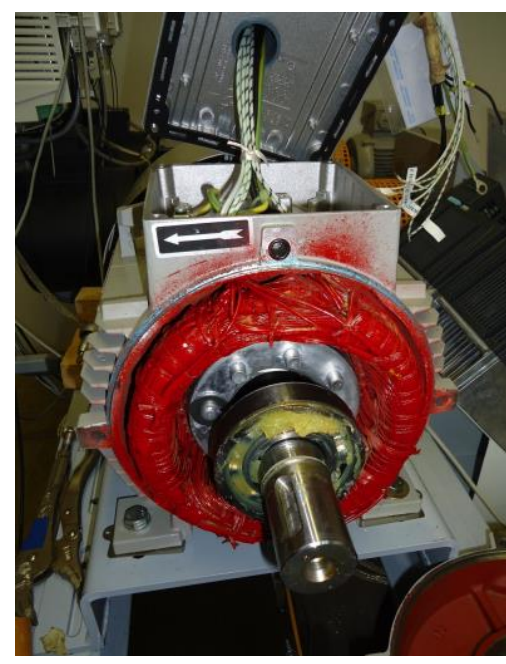

Figure 11. Disassembled motor exposing random would stator winding.

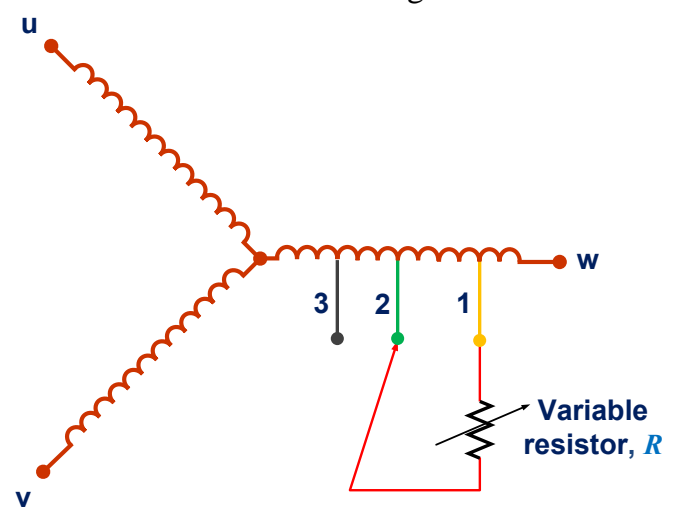

Figure 12. Schematic winding diagram with three taps on the phase $w$ winding for different inter-turn fault scenarios.

\begin{tabular}{ccccc}
\hline State & \multicolumn{2}{c}{ Inter-turn I } & \multicolumn{2}{c}{ Inter-turn II } \\
\cline { 2 - 5 } & $\boldsymbol{i}_{\boldsymbol{l}}[\mathbf{m A}]$ & $\boldsymbol{P}_{\boldsymbol{d}}[\mathbf{W}]$ & $\boldsymbol{i}_{\boldsymbol{l}}[\mathbf{m A}]$ & $\boldsymbol{P}_{\boldsymbol{d}}[\mathbf{W}]$ \\
\hline F1 & 265 & 40.7 & 86 & 4.3 \\
\hline F2 & 297 & 26.5 & 155 & 7.2 \\
\hline
\end{tabular}

Table 2. Current and dissipated power through the variable resistor at different states

\section{RESULTS AND DISCUSSION}

Under varying fault severity levels, squared envelope signal estimation was calculated by following the procedure introduced in Section 2.2.3. The result for healthy state, Interturn I and Inter-turn II in Test 1 are presented in Figure 13, respectively. Compared with the healthy state, it is obvious that the pattern of vibration of the induction motor has changed in time domain for inter-turn fault. The period of one cycle of vibration for the healthy case is 

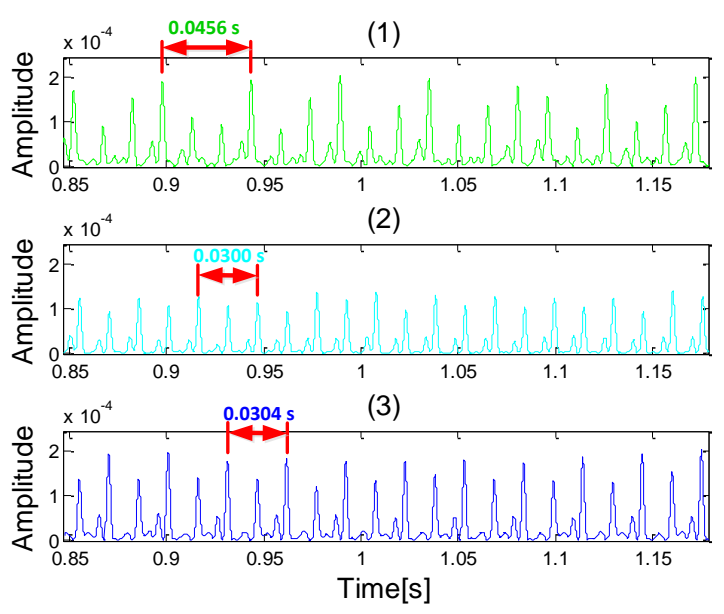

Figure 13. Time domain envelope signals for F1 in Test 1: (1) time domain envelope signal for healthy state with period of approx. $0.0456 \mathrm{~s}$, (2) time domain envelope signal for InterTurn I with period of approx. $0.0300 \mathrm{~s}$, (3) time domain envelope signal for Inter-Turn II with period of approx. $0.0304 \mathrm{~s}$.

approximately $0.0456 \mathrm{~s}$, and the period for both of the interturn cases is approximately $0.0300 \mathrm{~s}$, namely $33.3 \mathrm{~Hz}$ which is about the same with the rotational speed (2000 RPM/60 s= $33.3 \mathrm{~Hz}$ ). This is because inter-turn fault has changed the magnetic flux distribution of the induction motor and the faulty characteristic is related to the rotating speed. It is also noticeable that the amplitude of the faulty characteristic increases as the fault becomes more severe.

After obtaining the envelope signal, fast Fourier transform was applied with the DC component removed. For the purpose of comparing between different scenarios, amplitudes of the spectrum were "normalized" according to the DC component amplitude of each signal after Fourier transform, whose value should be dominant and the maximum in the whole spectrum; and the frequency domain was also transferred to order domain to emphasize the relationship of the harmonics and the rotational speed. In Figure 14, it is evident that at the first order there is a harmonic component for inter-turn faults. And by comparing (3) with (2) in Figure 14, the severity of the fault is also successfully revealed, with the more severe case possessing a higher value.

Furthermore, a bar plot was generated for all the conditions at different severity levels for both of the tests, which is shown in Figure 15. As one can observe, within one test, there is a clear difference between healthy state and inter-turn faults in terms of bar height. In terms of severity, for Interturn I and Inter-turn II respectively, amplitudes at F2 for Test 1 in (b) is larger than those for Test 1 in (a) of Figure 15. Besides, Inter-turn II has a larger value than
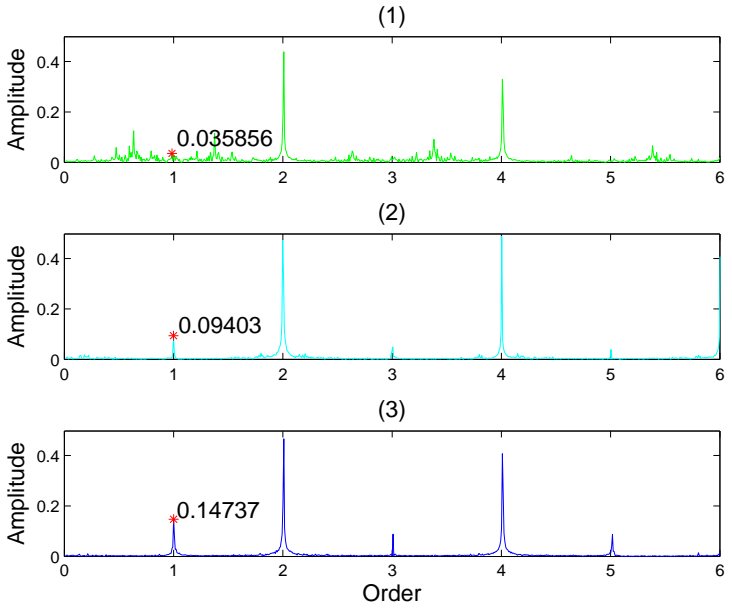

Figure 14. Envelope spectra in order domain for F1 in Test 1: (1) envelope spectrum for healthy state with no harmonic at the first order, (2) envelope spectrum for Inter-turn I with a peak valued at 0.09403 at the first order, (3) envelope spectrum for Inter-turn II with a peak valued at 0.14737 at the first order.
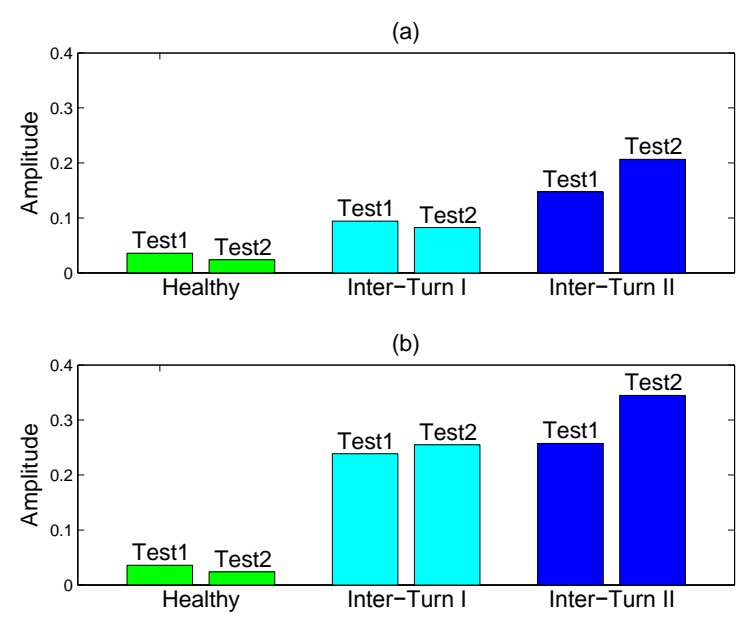

Figure 15. Amplitudes of first order component in envelope spectrum for different conditions and severity levels in the two tests: (a) amplitudes for all three conditions at severity level F1 of Test 1 and Test 2, (b) amplitudes for all three conditions at severity level F2 of Test 1 and Test 2 . The three colors represent healthy state, Inter-turn I, and Inter-turn II, respectively, which are consistent with previous figures.

Inter-turn I, which once again reveals the severity of fault successfully. Since the values of the order domain were normalized between 0 and 1 , it can be considered as a metric called hazard value (HV) to quantify inter-turn fault in induction motors. The result is shown in Table 3.

Test Healthy Inter-turn I $\quad$ Inter-turn II




\begin{tabular}{cccccc}
\hline & & F1 & F2 & F1 & F2 \\
\hline $\mathbf{1}$ & 0.0359 & 0.0940 & 0.2385 & 0.1474 & 0.2574 \\
\hline $\mathbf{2}$ & 0.0237 & 0.0822 & 0.2549 & 0.2064 & 0.3445 \\
\hline
\end{tabular}

Table 3. Hazard value (HV) of different conditions and severities

\begin{tabular}{cccccc}
\hline & Healthy & \multicolumn{2}{c}{ Inter-turn I } & \multicolumn{2}{c}{ Inter-turn II } \\
\cline { 3 - 5 } & & F1 & F2 & F1 & F2 \\
\hline $\begin{array}{c}\text { Difference } \\
(\%)\end{array}$ & 34.0 & 12.6 & 6.9 & 40.0 & 33.8 \\
\hline
\end{tabular}

Table 4. Consistency of tests. The percentage is calculated based on the differences between HVs in the two tests under the same condition.

\begin{tabular}{cccccc}
\hline $\begin{array}{c}\text { Test 1 } \\
(\%)\end{array}$ & Healthy & \multicolumn{2}{c}{ Inter-turn I } & \multicolumn{2}{c}{ Inter-turn II } \\
\cline { 3 - 6 } & & F1 & F2 & F1 & F2 \\
\hline Healthy & 0 & 161.8 & 564.3 & 310.6 & 617.0 \\
\hline $\begin{array}{c}\text { Inter-turn I- } \\
\text { F1 }\end{array}$ & - & 0 & 617.0 & 56.8 & 173.8 \\
\hline $\begin{array}{c}\text { Inter-turn I- } \\
\text { F2 }\end{array}$ & - & - & 0 & - & 7.9 \\
\hline $\begin{array}{c}\text { Inter-turn II- } \\
\text { F1 }\end{array}$ & - & - & - & 0 & 74.6 \\
\hline $\begin{array}{c}\text { Inter-turn II- } \\
\text { F2 }\end{array}$ & - & - & - & - & 0 \\
\hline
\end{tabular}

Table 5. Difference percentage between different conditions in Test 1. Compare the percentage increase in HV when the fault is getting more severe.

\begin{tabular}{cccccc}
\hline $\begin{array}{c}\text { Test } \\
(\%)\end{array}$ & Healthy & \multicolumn{2}{c}{ Inter-turn I } & \multicolumn{2}{c}{ Inter-turn II } \\
\cline { 2 - 6 } Healthy & 0 & 246.8 & 975.5 & 770.9 & $\begin{array}{c}1353 . \\
6\end{array}$ \\
\hline $\begin{array}{c}\text { Inter-turn I- } \\
\text { F1 }\end{array}$ & - & 0 & 210.1 & 151.1 & 319.1 \\
\hline $\begin{array}{c}\text { Inter-turn I- } \\
\text { F2 }\end{array}$ & - & - & 0 & - & 35.2 \\
\hline $\begin{array}{c}\text { Inter-turn II- } \\
\text { F1 }\end{array}$ & - & - & - & 0 & 67.0 \\
\hline $\begin{array}{c}\text { Inter-turn II- } \\
\text { F2 }\end{array}$ & - & - & - & - & 0 \\
\hline
\end{tabular}

Table 6. Difference percentage between different conditions in Test 2. Compare the percentage increase in HV when the fault is getting more severe.

In order to check the consistency and robustness of the result, comparisons of HVs between Test 1 and Test 2 are summarized in Table 4, Table 5, and Table 6. In Table 4, it can be seen that under the same experimental setting, the HV value can be different up to $40 \%$ from the previous test. This suggests that the robustness in the HV metric needs further improvement and the value might not serve as a universal measure for stator inter-turn faults. Nevertheless, we argue that the increasing trend of the feature when the fault is getting worse is more important than the value itself. From both Table 5 and Table 6 , it can be seen that each test provides a very distinctive HV for every faulty condition against the healthy state; and when the faulty level increases, HV also increases with it. This indicates the method still has the ability of detecting induction motor stator inter-turn faults, and measuring the degree of severity.

\section{CONCLUSION}

This paper proposes a vibration-based method to detect interturn winding fault, which is known to be the hardest to detect even with current and voltage signal. The method was divided into two stages, namely signal pre-processing stage and signal enhancement stage. In the pre-processing stage, data quality check and a low-pass filter were applied on both vibration signal and tachometer signal. In the signal enhancement stage, several techniques were adopted. Time synchronous averaging was used to remove the discrete frequency component noise, and then the residual signal was demodulated at the center frequency and bandwidth selected with the help of kurtogram. The resulting normalized envelope spectrum was converted into order domain, and the component at the first order was able to distinguish the interturn fault state from the healthy state, and at the same time reflect the severity.

There is certainly space for improvement when looking at the robustness and generality of the method. This method is applied at a constant speed and constant load condition. Whether or not the method could be applied at transient circumstances needs further study and validation. Besides, the proposed HV might not be able to serve as a general measure for stator inter-turn fault severity, because the variations might be high and nonlinear. Instead, it is suggested that the HVs be used as "features" that are able to distinguish healthy states from faulty and capture the trendy change of the inter-turn fault development. Then the features can be fed into other more advanced machine learning algorithms to determine the condition of the motor.

In terms of removing the discrete frequency component, TSA is somewhat a computationally expensive method. To achieve similar effects, cepstrum analysis can serve as another option (Randall \& Sawalhi, 2011). Cepstrum usually would be applied to separate the transmission noise from the sources, and is easier to implement since it is based on the computation of fast Fourier transform, which is very efficient. The only drawback of cepstrum analysis is that the deterministic component frequencies need to be known a prior. So in cases of enough domain knowledge about the induction motor, cepstrum could be a superior choice. 


\section{ACKNOWLEDGEMENT}

Authors of the NSF I/UCRC for Intelligent Maintenance Systems (IMS) at the University of Cincinnati would like to acknowledge the generous support from Flanders' Mechatronics Technology Centre (FMTC), a division of Flanders' Make vzw, for this collaborative research effort to move forward the technologies of induction motor prognostics.

\section{REFERENCES}

Al-Atat, H., Siegel, D. \& Lee, J. (2011). A systematic methodology for gearbox health assessment and fault classification. Int J Prognostics Health Manage Soc, vol. 2(1), pp. 16.

Antoni, J. (2007). Fast computation of the kurtogram for the detection of transient faults. Mechanical Systems and Signal Processing, vol. 21(1), pp. 108-124.

Antoni, J. \& Randall, R. (2006). The spectral kurtosis: application to the vibratory surveillance and diagnostics of rotating machines. Mechanical Systems and Signal Processing, vol. 20(2), pp. 308-331.

Bechhoefer, E. \& Kingsley, M. (2009). A review of time synchronous average algorithms. Annual conference of the prognostics and health management society

Bell, R., McWilliams, D., O'Donnell, P., Singh, C. \& Wells, S. (1985). Report of large motor reliability survey of industrial and commercial installations. I. IEEE Transactions on Industry Applications, vol. 21(4), pp. 853-864.

Cardoso, A. M., Cruz, S. \& Fonseca, D. (1999). Inter-turn stator winding fault diagnosis in three-phase induction motors, by Park's vector approach. Energy Conversion, IEEE Transactions on, vol. 14(3), pp. 595-598.

Djurović, S., Vilchis-Rodriguez, D. S. \& Smith, A. C. (2014) Investigation of wound rotor induction machine vibration signal under stator electrical fault conditions. The Journal of Engineering.

Eftekhari, M., Moallem, M., Sadri, S. \& Hsieh, M.-F. (2013). Online Detection of Induction Motor's Stator Winding Short-Circuit Faults. Systems Journal, IEEE, vol. 8(4), pp. 1272 - 1282. doi: 10.1109/JSYST.2013.2288172

Furfari, F. \& Brittain, J. (2002). Charles LeGeyt Fortescue and the method of symmetrical components. Industry Applications Magazine, IEEE, vol. 8(3), pp. 7-9.

Jablonski, A. \& Barszcz, T. (2013). Validation of vibration measurements for heavy duty machinery diagnostics. Mechanical Systems and Signal Processing, vol. 38(1), pp. 248-263.

Jabłoński, A., Barszcz, T. \& Bielecka, M. (2011). Automatic validation of vibration signals in wind farm distributed monitoring systems. Measurement, vol. 44(10), pp. 1954-1967.

Jardine, A. K., Lin, D. \& Banjevic, D. (2006). A review on machinery diagnostics and prognostics implementing condition-based maintenance. Mechanical Systems and Signal Processing, vol. 20(7), pp. 1483-1510.

Jover Rodríguez, P. V. \& Arkkio, A. (2008). Detection of stator winding fault in induction motor using fuzzy logic. Applied Soft Computing, vol. 8(2), pp. 1112-1120.

Kliman, G., Premerlani, W., Koegl, R. \& Hoeweler, D. (1996). A new approach to on-line turn fault detection in AC motors. Industry Applications Conference, 1996. Thirty-First IAS Annual Meeting, IAS'96., Conference Record of the 1996 IEEE (687-693)

Kliman, G. \& Stein, J. (1992). Methods of motor current signature analysis. Electric Machines and power systems, vol. 20(5), pp. 463-474.

Kohler, J. L., Sottile, J. \& Trutt, F. C. (2002). Condition monitoring of stator windings in induction motors. I. Experimental investigation of the effective negativesequence impedance detector. Industry Applications, IEEE Transactions on, vol. 38(5), pp. 1447-1453.

Lamim Filho, P., Pederiva, R. \& Brito, J. (2014). Detection of stator winding faults in induction machines using flux and vibration analysis. Mechanical Systems and Signal Processing, vol. 42(1), pp. 377-387.

Lamim, P., Brito, J. N., Silva, V. A. D. \& Pederiva, R. (2013). Detection of Electrical Faults in Induction Motors Using Vibration Analysis. Journal of Quality in Maintenance Engineering, vol. 19(4), pp. 2-2.

Lin, J. \& Qu, L. (2000). Feature extraction based on Morlet wavelet and its application for mechanical fault diagnosis. Journal of sound and vibration, vol. 234(1), pp. 135-148.

Nandi, S., Toliyat, H. A. \& Li, X. (2005). Condition monitoring and fault diagnosis of electrical motors-a review. Energy Conversion, IEEE Transactions on, vol. 20(4), pp. 719-729.

Ompusunggu, A. P., Liu, Z., Ardakani, H. D., Jin, C., Petre, F. \& Lee, J. (2014). Winding fault diagnosis of a 3-phase induction motor powered by frequency-inverter drive using the current and voltage signals. Proceedings of the 14th Mechatronics Forum International Conference. June 16-18, Karlstad (Sweden)

Randall, R. B. \& Antoni, J. (2011). Rolling element bearing diagnostics - a tutorial. Mechanical Systems and Signal Processing, vol. 25(2), pp. 485-520.

Randall, R. B. \& Sawalhi, N. (2011). A new method for separating discrete components from a signal. Sound and Vibration, vol. 45(5), pp. 6.

Seshadrinath, J., Singh, B. \& Panigrahi, B. K. (2014). Investigation of Vibration Signatures for Multiple Fault Diagnosis in Variable Frequency Drives Using Complex Wavelets. Power Electronics, IEEE Transactions on, vol. 29(2), pp. 936-945.

Siegel, D., Al-Atat, H., Shauche, V., Liao, L., Snyder, J. \& Lee, J. (2012). Novel method for rolling element bearing health assessment-A tachometer-less synchronously averaged envelope feature extraction technique. 
Mechanical Systems and Signal Processing, vol. 29, pp. 362-376.

Siegel, D., Ly, C. \& Lee, J. (2012). Methodology and framework for predicting helicopter rolling element bearing failure. Reliability, IEEE Transactions on, vol. 61(4), pp. 846-857.

Sin, M. L., Soong, W. L. \& Ertugrul, N. (2003). Induction machine on-line condition monitoring and fault diagnosis - a survey. Australasian Universities Power Engineering Conference (1-6), Christchurch, New Zealand

Sottile, J., Trutt, F. C. \& Kohler, J. L. (2000). Experimental investigation of on-line methods for incipient fault detection [in induction motors]. Industry Applications Conference (2682-2687)

Trutt, F. C., Sottile, J. \& Kohler, J. L. (2002). Online condition monitoring of induction motors. Industry Applications, IEEE Transactions on, vol. 38(6), pp. 1627-1632.

Ukil, A., Chen, S. \& Andenna, A. (2011). Detection of stator short circuit faults in three-phase induction motors using motor current zero crossing instants. Electric Power Systems Research, vol. 81(4), pp. 1036-1044. 